\title{
A Fuzzy Method for Joint Resource Allocation and Stable Pairing in D2D Communications with Applications in COVID-19 Patient Information Management
}

\author{
Alireza Pourmoslemi \\ Payame Noor University \\ Siavash Rajabi ( $\nabla$ siavash.rajabi@hut.ac.ir) \\ Hamedan University of Technology \\ Mehdi Salimi \\ McMaster University \\ Massimiliano Ferrara \\ UniversitàUniversit'Università Mediterranea di Reggio Calabria
}

\section{Research Article}

Keywords: device-to-device (D2D), Long-Term Evolution (LTE), network infrastructure, equipment

Posted Date: July 22nd, 2021

DOI: https://doi.org/10.21203/rs.3.rs-728511/v1

License: (c) (1) This work is licensed under a Creative Commons Attribution 4.0 International License.

Read Full License 


\title{
A Fuzzy Method for Joint Resource Allocation and Stable Pairing in D2D Communications with Applications in COVID-19 Patient Information Management
}

\author{
Alireza Pourmoslemi ${ }^{1}$, Siavash Rajabi ${ }^{2, *}$, Mehdi Salimi $^{3,4}$, and Massimiliano Ferrara ${ }^{5}$ \\ ${ }^{1}$ Department of Mathematics, Payame Noor University, P.O.BOX 19395-3697, Tehran, Iran \\ ${ }^{2}$ Department of Electrical Engineering, Hamedan University of Technology, Hamedan 65155, Iran \\ ${ }^{3}$ Department of Mathematics \& Statistics, McMaster University, Hamilton, Ontario, Canada \\ ${ }^{4}$ Center for Dynamics, Faculty of Mathematics, Technische Universität Dresden, Dresden, Germany \\ ${ }^{5}$ DiGiES \& Decisions Lab, Università Mediterranea di Reggio Calabria, Reggio Calabria, Italy \\ *siavash.rajabi@hut.ac.ir
}

\begin{abstract}
In this paper, a device-to-device (D2D) communication multiple-metric scenario for resource allocation and pairing is proposed. To improve the stability of the D2D connection, a fuzzy method based on the data-rate and battery levels of potential D2D pairs is studied so that the set of D2D transmitters is considered as a fuzzy set. A single-cell scenario with a cellular user and some co-channel D2D nodes consisting of D2D receivers and transmitters is considered. A stable fuzzy pairing criterion is proposed for the selection of the best D2D transmitter. The proposed method is compared to three other pairing methods: maximum sum-rate, constant, and random pairing methods. The simulation results show that the proposed pairing method outperforms the other three methods in terms of stability and fairness criteria and follows the maximum sum-rate method from the sum-rate criteria point of view.
\end{abstract}

\section{Introduction and Related Works}

The increase in the number of wireless network users and applications has led to an increase in the demand for cellular network resources. This challenge has led to the formation of services that reduce the load on the central part of the network. Device-to-device (D2D) communication is a proximity-based service that enables two devices to exchange information without the need for a base station, and is standardized by 3GPP Long-Term Evolution (LTE). D2D communication is divided into overlay in-band, underlay in-band, and out-band communications. With in-band communications, D2D users utilize the cellular network spectrum that is categorized into overlay and underlay ${ }^{1}$.

The underlay in-band is a D2D communication mode that allows the D2D users to use the cellular spectrum and causes interference with cellular links and vice versa. The cellular spectrum is distributed between cellular and D2D users in overlay in-band mode with no interference. In out-band communications, users use a different spectrum than the cellular spectrum. The main advantages of D2D service are the optimal allocation of cellular network resources, power consumption, cellular network traffic offloading, and the possibility of creating public safety networks ${ }^{2}$.

Recently, with the spread of viral diseases, especially coronavirus, there is a need to use more decentralized networks. The rapid spread of COVID-19 has changed lifestyles and increased people's tendency to perform activities remotely. Under these conditions, the need to connect to communication networks has increased. Moreover, the increase in the number of patients in hospitals has led to hospital network resources generally not meeting the need for treatment during the pandemic. Therefore, services that can shift the focus away from cellular and centralized networks will be of particular importance. Suppose, for example, a hospital that receives a large number of patients but the network communication infrastructure to transfer patient data to physicians is limited. In this case, many problems will be solved if large amounts of medical data can be transferred without using the central network infrastructure. D2D service can be one of the options to overcome these challenges. In this article, considering the two main goals, sustainable communication and the transmission of bulky contents, a scenario of joint resource allocation and pairing in a decentralized network based on D2D communication is studied.

One of the applications of the mentioned scenario is described as follows: Imagine a hospital with a large number of coronary patients while the network infrastructure, equipment, and staff of the hospital are limited to serve all patients. If physicians 
connect to the centralized network of the hospital to check patient data such as CT scans, MRIs, large EEG data, etc., this increases the possibility of network interruption. We claim that the here-proposed method can solve the mentioned problem well. In the following, we explain the proposed method and then describe the mentioned hospital.

\subsection{Pairing in D2D Communications}

In the history of D2D communications, it is often supposed that D2D pairs are formed in advance. The main issues are selecting the proper cellular user with whom to share resources, selecting the operating mode, and setting the power for cellular and D2D users. In the literature, pairing is defined and investigated as permitting a D2D connection (a transmitter and its associated receiver) to use the resource of a cellular connection ${ }^{3-6}$. However, stable pairing for a D2D user in a practical scenario remains an essential problem.

This problem is investigated and studied in various approaches in the literature ${ }^{7-13}$. The nearest transmitter is considered to be the best transmitter in ${ }^{7-10}$, but the nearest transmitter is not necessarily the best ${ }^{11-13}$. Our approach to pairing is similar to those of ${ }^{11-13}$, except that we consider the problem of multi-user pairing and resource allocation.

\subsection{Fuzzy Mathematics}

Zadeh introduced fuzzy sets in his significant paper ${ }^{14}$ in 1965 . His idea was to introduce a generalization of the concept crisp sets. The idea was to use numbers in $[0,1]$ as the membership degrees of elements instead of 0 and 1.

Definition 0.1. ${ }^{15}$ Suppose that $X$ is a collection of objects. Then, $A=\{(x, \mu(x)): x \in X\}$ is called a fuzzy subset of $X$, where $\mu: X \rightarrow[0,1]$ is a function called the membership function, which denotes the membership degrees of elements in $X$.

In 1966, an application of fuzzy sets to find a pattern classification was introduced by Bellman et al. ${ }^{16}$. Four years later, a decision-making problem was solved by Zadeh and Bellman ${ }^{17}$. In the 1970s and 1980s, decision making, control, and pattern classification received the most attention by researchers ${ }^{18-20}$. The fuzzy mathematics applications were expanded in the next years. Another important application of fuzzy sets is in fuzzy controllers ${ }^{21}$. Fuzzy genetics algorithms and neuro-fuzzy systems were studied in the $1990 \mathrm{~s}^{22}$. Pattern recognition, machine learning, and wireless sensor networks are other fields of study that have benefited from fuzzy mathematics ${ }^{22-24}$.

Fuzzy mathematics in D2D communications have mostly been applied in the field of resource allocation and power control. A continuous fuzzy power control scheme was studied by ${ }^{25}$, so that the cellular user can communicate in low power to achieve better D2D user connection quality. A resource allocation algorithm is modeled through fuzzy mathematics and game theory $\mathrm{in}^{26}$ to introduce an uncertain relationship between the resource and user. $\mathrm{In}^{27}$, a fuzzy clustering algorithm is proposed to divide D2D users into several groups to reuse the cellular user resources and repress co-channel interference. In ${ }^{28}$, a resource allocation based on fuzzy clustering is proposed to solve the spectrum scarcity problem. A fuzzy-logic-based vehicle handover algorithm is studied in ${ }^{29}$ to select the proper access network.

In this paper, we consider each transmitter as a fuzzy node in a D2D mobile network so that a set of transmitters together with a membership function is regarded as a fuzzy set. The membership function assigns a number in $[0,1]$ to each transmitter based on the transmitter's data-rate and battery level. A decision-making function then chooses the best transmitter with the highest fuzzy degree.

We use triangular norms and co-norms to build our fuzzy membership function.

Definition 0.2. ${ }^{30}$ Let $T:[0,1] \times[0,1] \rightarrow[0,1]$ be a commutative, associative, and non-decreasing function, which $T(x, 1)=x$, for all $x \in[0,1]$. Then, $T$ is called a triangular norm ( $t$-norm).

For example, basic $t$-norms that will be called Lukasiewicz, Minimum, and Product $t$-norms are defined as follows, respectively:

1. $T_{L}(x, y)=\max (x+y-1,0)$,

2. $T_{M}(x, y)=\min (x, y)$, and

3. $T_{P}(x, y)=x \cdot y$.

Schweizer and Sklar introduced $t$-conorms as dual operations of $t$-norms in $1961^{31}$.

Definition 0.3. ${ }^{30}$ Let $S:[0,1] \times[0,1] \rightarrow[0,1]$ be an associative, commutative, and monotone function that $S(x, 0)=$ $x$ for all $x \in[0,1]$. Then, $S$ is called a triangular conorm ( $t$-conorm).

Correspond to basic $t$-norms, there are some $t$-conorms written as follows: 
1. $S_{L}(x, y)=\min (x+y, 1)$, (Lukasiewicz t-conorm),

2. $S_{M}(x, y)=\max (x, y)$, (Maximum $t$-conorm),

3. $S_{P}(x, y)=x+y-x \cdot y$, (Probabilistic sum).

Another $t$-conorm, named the Einstein sum, is used in this paper to build our membership function ${ }^{30}$. The Einstein sum $\tau:[0,1] \times[0,1] \rightarrow[0,1]$ is defined as follows for all $x, y \in[0,1]:$

$$
\tau(x, y)=\frac{x+y}{1+x y} .
$$

To find a proper membership function, the Einstein sum is chosen after examining several $t$-norms and $t$-conorms. Other suitable triangular norms and conorms can be examined in future studies. For more details about the applications of the Einstein sum in fuzzy systems, $\operatorname{see}^{32-34}$.

\subsection{Contributions}

The main contributions of this paper are summarized as follows:

1. A joint resource allocation and pairing scenario in D2D communications is investigated. A number of D2D receivers are considered for the reuse of cellular network resources. For each D2D receiver, there exist a number of potential D2D transmitters and a stable fuzzy pairing criteria is proposed for the selection of the best transmitter.

2. The set of transmitters is considered as a fuzzy set so that a fuzzy degree is assigned to each node concerning the data-rates and battery levels in connection with their potential receiver. We claim that this fuzzy membership function can be considered as a measure of the stability of the connections.

3. To examine the proposed method, it is compared with three other pairing methods, so that the three parameters of stability, fairness, and sum-rate in different modes, such as changing the D2D search radius, increasing the number of D2D transmitters, and receivers for all four methods, are investigated.

4. The proposed method for D2D pairing, in addition to reducing the traffic load of the cellular network, leads to more stable connections with a higher quality of service. One of the significant applications of this method is that bulky content can be transferred more reliably.

\section{System Model}

In this paper, we consider the problem of joint resource allocation and stable pairing in a D2D-enabled cellular communication network. The system model consists of a single cell with a cellular user and a number of co-channel D2D nodes. Some D2D nodes are transmitters and some are receivers, so that there are several potential transmitters for each receiver. The transmitters of each receiver are specified separately to share different contents (Fig. 1). We tend to find the most suitable transmitter for pairing with each receiver so that stability is maintained. By stability, we mean a situation in which the battery levels and the data-rates of two potential paired nodes are acceptable. For this purpose, considering potential transmitting D2D users as fuzzy nodes, the normalized smartphone battery levels and data-rates are used to obtain an index for stability.

Let $\mathscr{D}_{r}=\left\{r_{1}, r_{2}, \ldots, r_{N}\right\}$ be the set of D2D receivers, where $N \in \mathbb{N}$ is the maximum number of co-channel D2D receivers with a cellular user in the network. For each D2D receiver $r_{i}(i=1, \ldots, N)$, the set $\mathscr{D}_{t_{i}}=\left\{t_{1 i}, t_{2 i}, \ldots, t_{K i}\right\}$ denotes the potential $\mathrm{D} 2 \mathrm{D}$ transmitters, where $K \in \mathbb{N}$ is the number of D2D transmitters around the receiver $r_{i}$. Moreover, the D2D transmitters are located in a circle centered on each D2D receiver with radius $R_{\max }$, where $R_{\max }$ is the maximum search radius of D2D receivers.

\subsection{Communication Models}

The system model channels are supposed to be independent and identically distributed (i.i.d.) Rayleigh channels with perfect channel state information (CSI), only available for D2D receivers mentioned as follows:

1. $h_{c, B}$ and $d_{c, B}$ are the direct channel and distance between the cellular user and evolved node base station (eNB), respectively.

2. $h_{t_{k i}, r_{j}}$ and $d_{t_{k i}, r_{j}}$ are the channel and distance between the D2D transmitter $t_{k i}$ and the D2D receiver $r_{j}$, respectively, where $t_{k i} \in \mathscr{D}_{t_{i}}, r_{j} \in \mathscr{D}_{r}, k \in\{1, \ldots, K\}$, and $i, j \in\{1, \ldots, N\}$. 


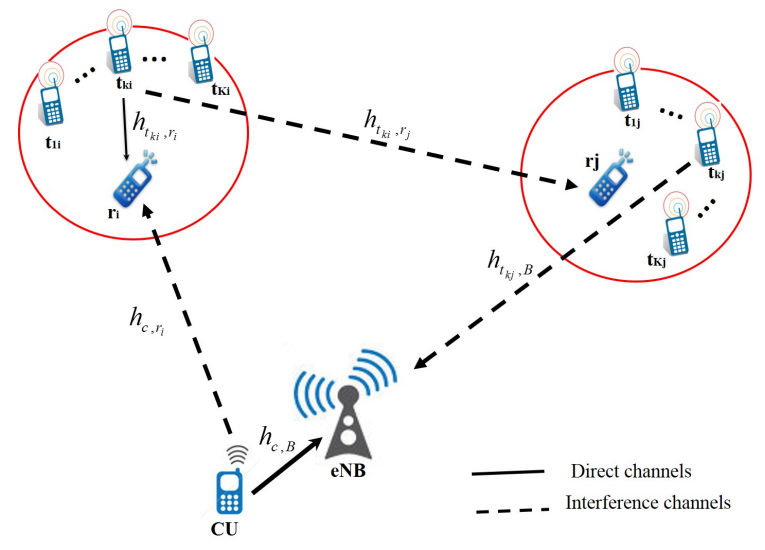

Figure 1. System model.

3. $h_{t_{k i}, B}$ and $d_{t_{k i}, B}$ are the interference channel and distance between the D2D transmitter $t_{k i}$ and eNB, respectively.

4. $h_{c, r_{i}}$ and $d_{c, r_{i}}$ are the interference channel and distance between the cellular user and D2D receiver $r_{i}$, respectively.

The cellular and D2D links' SINR (Signal to Interference plus Noise Ratio) can be noted as follows:

$$
\begin{aligned}
& \operatorname{SINR}_{B}=\frac{S_{B}}{I_{B}+N_{0}}, \\
& \operatorname{SINR}_{k i}=\frac{S_{k i}}{I_{i}+N_{0}},
\end{aligned}
$$

where

$$
\begin{gathered}
S_{B}=P^{c}\left|h_{c, B}\right|^{2}\left|d_{c, B}\right|^{-\alpha}, \\
S_{k i}=P^{d} \beta_{k i}\left|h_{t_{k i}, r_{i}}\right|^{2}\left|d_{t_{k i}, r_{i}}\right|^{-\alpha}, \\
I_{B}=\sum_{j=1}^{N} \sum_{k=1}^{K} \beta_{k j} P^{d}\left|h_{t_{k j}, B}\right|^{2}\left|d_{t_{k j}, B}\right|^{-\alpha}, \\
I_{i}=\sum_{j=1}^{N} \sum_{k^{\prime}=1}^{K} \beta_{k^{\prime} j} P^{d}\left|h_{t_{k^{\prime} j}, r_{i}}\right|^{2}\left|d_{t_{k^{\prime} j}, r_{i}}\right|^{-\alpha}+P^{c}\left|h_{c, r_{i}}\right|^{2}\left|d_{c, r_{i}}\right|^{-\alpha} .
\end{gathered}
$$

Note that $N_{0}$ is the received noise power and $\beta_{k j}$ is the binary pairing variable denoted by

$$
\beta_{k j}= \begin{cases}1, & \text { if } t_{k j} \text { is paired with the receiver } r_{j} ; \\ 0, & \text { otherwise. }\end{cases}
$$

According to the above SINR denotations, the cellular and D2D user data-rates are written, respectively, as

$$
\begin{aligned}
& R_{B}=\log \left(1+\operatorname{SINR}_{B}\right), \\
& R_{k i}=\log \left(1+\operatorname{SINR}_{k i}\right) .
\end{aligned}
$$




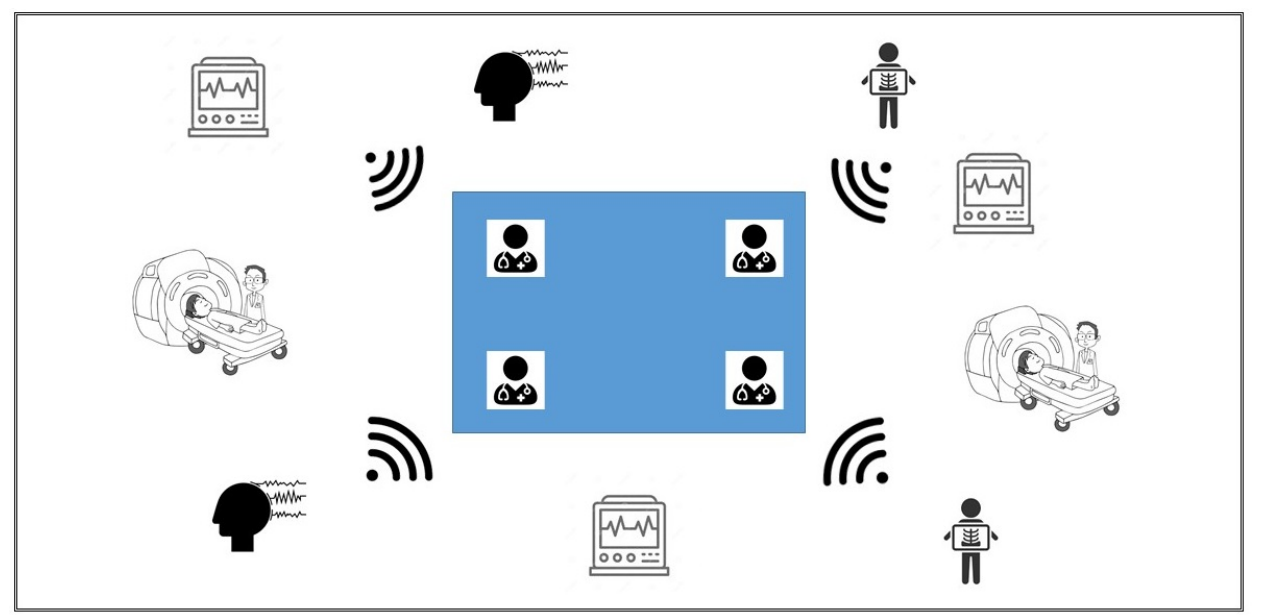

Figure 2. Hospital equipped with a decentralized network.

\subsection{Constructing the fuzzy membership function}

In the mentioned network, a stable connection is a connection in which the data-rate is acceptable and the link does not disconnect due to a low battery condition. The set of all nodes suitable for pairing has a fuzzy interpretation. Let the set $T=\left\{t_{k i} \mid t_{k i}\right.$ is a stable transmitter $\}$ be the fuzzy set, where $k \in\{1, \ldots, K\}$ and $i \in\{1, \ldots, N\}$. We construct a fuzzy membership function for each node in $T$. Two network parameters, the normalized data-rate $(R)$ and battery level $(E)$ of each node, are considered. To measure the stability, two thresholds, $R_{0}$ and $E_{0}$, are set. Considering the limitations of the network protocols, these thresholds guarantee the stability of the D2D link.

Concerning the mentioned explanations, the proposed membership function $\mu: T \rightarrow[0,1]$ is defined as follows:

$$
\mu\left(t_{k i}\right)=\lambda\left(R_{k i}, E_{k i}\right),
$$

where

$$
\lambda\left(R_{k i}, E_{k i}\right)= \begin{cases}R_{k i} ; & \left(E_{0} \leq E_{k i} \leq 1,\right. \\ & \left.0 \leq R_{k i}<R_{0}\right) \\ E_{k i} ; & \left(0 \leq E_{k i}<E_{0},\right. \\ \tau\left(R_{k i}, E_{k i}\right)\left(R_{k i}^{2}+E_{k i}^{2}\right) ; & \left(0<R_{k i} \leq 1\right) \\ & \left.0<R_{k i}<R_{0}\right) \\ \tau\left(R_{k i}, E_{k i}\right) ; & \left(E_{0} \leq E_{k i} \leq 1,\right. \\ & \left.R_{0} \leq R_{k i} \leq 1\right) .\end{cases}
$$

Note that the Einstein sum is applied as

$$
\tau\left(R_{k i}, E_{k i}\right)=\frac{R_{k i}+E_{k i}}{1+R_{k i} E_{k i}} .
$$

Because the fuzzy nodes with a high membership degree have both a higher battery level and a higher data-rate among their neighbors, the proposed membership function is an appropriate metric for measuring stability. For this reason, when transferring high-volume contents, the connection is less likely to fail. One of the applications of these stable connections is shown in Fig. 2. In this hospital with a decentralized D2D network, the physicians shown in the center of the figure use D2D links to access data shared by equipment and hospital staff while optimally using network resources.

\section{Simulation Results}

In this section, four different criteria for pairing in a D2D communication scenario are investigated. These four methods are:

- Fuzzy-based pairing (FP);

- Max sum-rate pairing (MSP); 
Table 1. Simulation parameters

\begin{tabular}{|c|c|}
\hline Parameter & Value \\
\hline$R_{c}$ (cellular radius) & $500 \mathrm{~m}$ \\
\hline$R_{\max }$ (D2D search radius) & $200 \mathrm{~m}$ \\
\hline $\begin{array}{l}\mathrm{N} \text { (The number of D2D receivers } \\
\text { for each cellular user) }\end{array}$ & $1-4$ \\
\hline $\begin{array}{l}\mathrm{K} \text { (The number of D2D transmitters } \\
\text { for each receiver) }\end{array}$ & $1-4$ \\
\hline$P^{c}$ (Cellular user's power) & $23 \mathrm{dBm}$ \\
\hline$P^{d}$ (D2D transmitters' power) & $15 \mathrm{dBm}$ \\
\hline$\alpha$ (path loss exponent) & 3.5 \\
\hline$R_{0}$ (Data-rate threshold) & 0.3 \\
\hline$E_{0}$ (Battery level threshold) & 0.25 \\
\hline
\end{tabular}

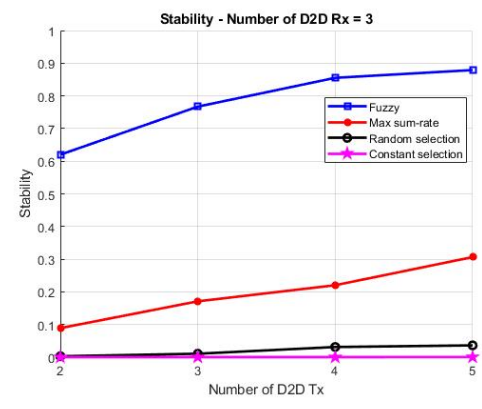

(a)

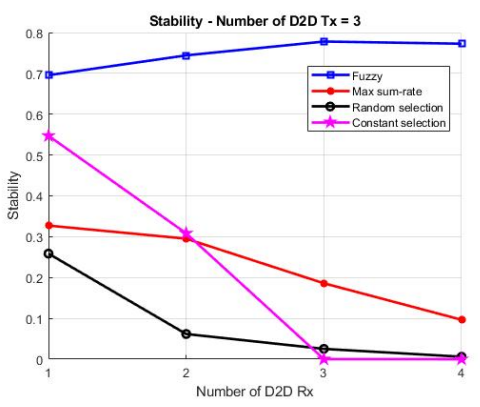

(b)

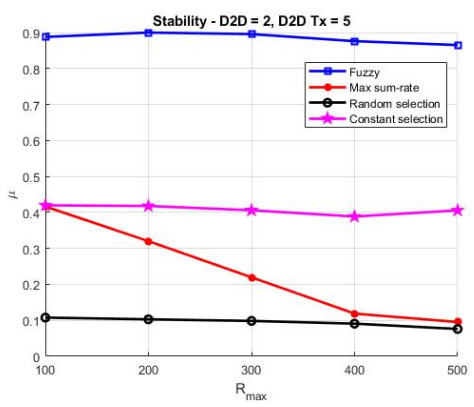

(c)

Figure 3. The stability as a function of (a) the number of potential D2D transmitters, (b) the number of co-channel D2D receivers, and (c) the $\mathrm{D} 2 \mathrm{D}$ search radius.

- Random pairing (RP);

- Constant pairing (CP).

In the MSP method, the desired transmitter for D2D communication is selected based on the highest sum-rate. According to (1.3) and (1.4), the sum-rate of the co-channel cellular and potential D2D pairs is calculated as $R_{B}+\sum_{i=1}^{N} R_{k i}$. In the RP method, the D2D transmitters are selected randomly. Finally, the nearest transmitter is considered the paired transmitter in the CP method. The main simulation parameters are summarized in Table (1).

Now, we compare the pairing stability measured by (1.5) for all four mentioned methods. Fig. 3 compares the stability as a function of the number of potential D2D transmitters around a receiver, the number of co-channel D2D receivers, and D2D search radius. In Fig. 3a, the pairing stability in the FP method increases considerably when the number of potential transmitters around a receiver increases. The pairing stability increase of the MSP method is much less than that of the FP method, whereas in the CP and RP methods, this increase is negligible. In FP and MSP methods, increasing the number of potential transmitters increases the number of proper choices for the receiver.

Fig. $3 b$ illustrates the stability of all four methods. The number of the D2D transmitters is three, and the number of receivers increases from one to four. Increasing the number of D2D receivers results in maintaining the stability of the FP method, but the stability decreases sharply in the other three methods.

In Fig. 3c, for a fixed number of D2D transmitters and receivers, the stability associated with increasing the search radius is calculated for all mentioned methods. Increasing the search radius does not significantly affect the stability of the FP, CP, and RP methods; however, it reduces the stability of the MSP method. The stability of the MSP method decreases with increasing the $R_{\max }$ for two reasons: first, the users' data-rates decrease with increasing $R_{\max }$; second, the battery levels are not considered in this method.

In Fig. 4, Jain's fairness index is computed for the number of potential D2D transmitters around a receiver, the number of 


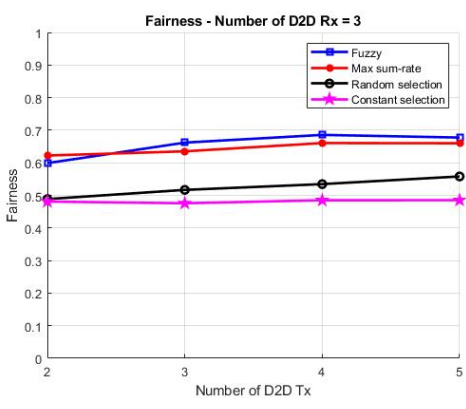

(a)

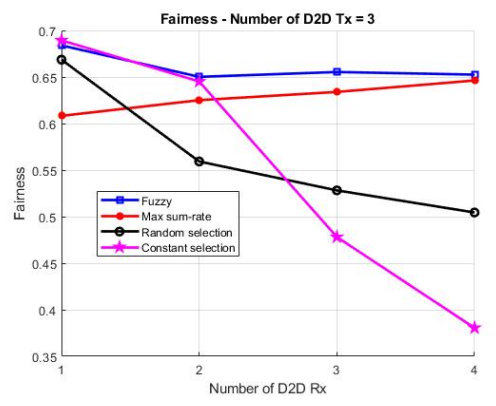

(b)

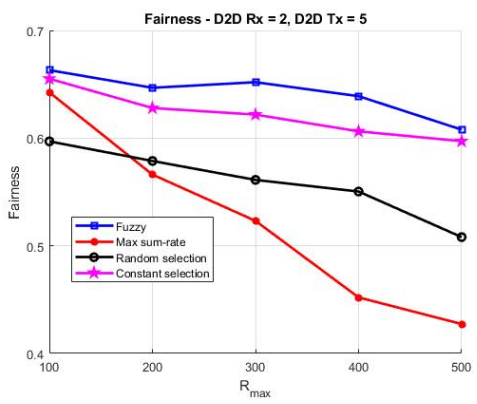

(c)

Figure 4. The Jain's fairness index compared according to (a) the number of potential D2D transmitters, (b) the number of co-channel D2D receivers, and (c) the D2D search radius.

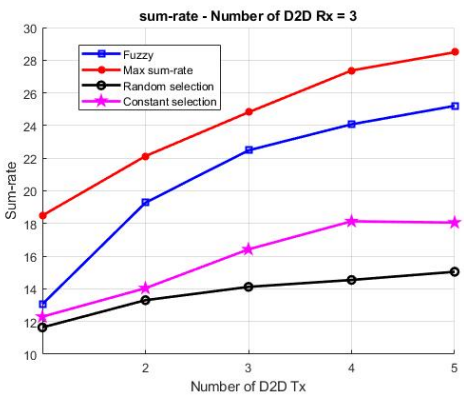

(a)

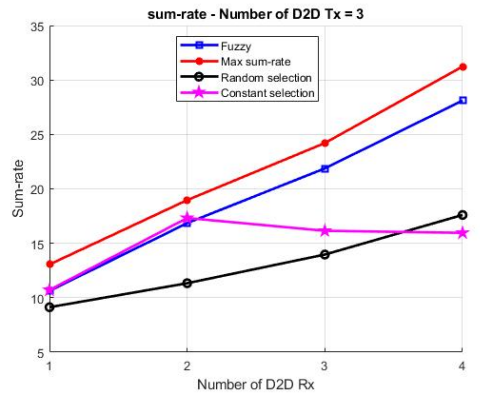

(b)

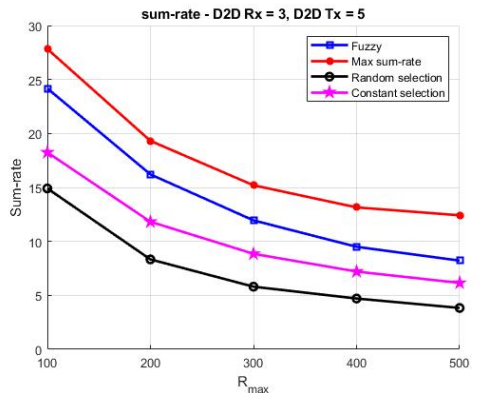

(c)

Figure 5. The sum-rate as a function of (a) the number of potential D2D transmitters, (b) the number of co-channel D2D receivers, and (c) the D2D search radius.

co-channel D2D receivers, and the D2D search radius. Jain's fairness index can be computed as follows:

$$
\mathscr{J}\left(R_{1}, R_{2}, \ldots, R_{N}\right)=\frac{\left(\sum_{i=1}^{N} R_{i}\right)^{2}}{N \cdot \sum_{i=1}^{N} R_{i}^{2}} .
$$

Fig. 4 illustrates that the FP method fairness index is maintained for all methods in a constant range, regardless of whether the number of receivers or transmitters or the D2D search radius increase. The fairness index in the MSP method behaves similarly to the FP method when $R_{\max }$ is constant. Nevertheless, when increasing $R_{\max }$, the fairness index of the MSP method decreases sharply. By increasing $R_{\max }$, the range of changes in the data-rate of co-channel users also increases and causes a reduction in the fairness index.

Increasing the number of transmitters has little effect on the fairness index of both CP and RP methods, but increasing the number of receivers reduces the fairness index of both methods. The reason is the broader range of users' data-rates. Furthermore, the fairness index of the CP and RP methods reduces slightly with the increase in D2D search radius.

Finally, Fig. 5 compares the sum-rate as a function of the number of potential D2D transmitters around a receiver, the number of co-channel D2D receivers, and D2D search radius. In Fig. 5a, the sum-rates of all four methods are compared when the number of transmitters is increased. The MSP method has the highest data-rate. The FP method is made similar to the MSP method by increasing the number of potential transmitters. The other two methods (RP and CP) do not follow the MSP method in this case.

In Fig. 5b, similar to Fig. 5a, increasing the number of D2D receivers, the FP method sum-rate follows the MSP method and the sum-rate of the RP method increases slowly, but after increasing the number of receivers from three to four, due to the effect of interference on the rate, the sum-rate of the $\mathrm{CP}$ method tends to a constant value.

In Fig. 5c, the sum-rate is plotted as a function of the D2D search radius for the four methods. As expected, due to the increased interference, the sum-rate decreases in all four methods as the search radius increases. However, the FP method is still the closest method to the MSP method. 


\section{Conclusions}

This paper proposed a new method for joint multi-pairing and resource allocation in a single-cell D2D scenario. Our method is based on fuzzy pairing criteria for the selecting the best transmitter in a D2D receiver search radius. The set of transmitters is considered as a fuzzy set with respect to the data-rates and battery levels of the transmitters. This fuzzy membership function can be considered as a stability index. The proposed method was compared with three other pairing methods: MSP, CP, and RP. The three parameters of stability, fairness, and sum-rate in different modes, i.e., changing the D2D search radius, increasing the number of D2D transmitters, and receivers for all four methods, were investigated. As a result, the fairness and stability of the fuzzy method are better than those of the other methods in different modes, and the FP method's sum-rate follows the MSP method's sum-rate.

\section{References}

1. Mach, P., Becvar, Z. \& Vanek, T. In-band device-to-device communication in ofdma cellular networks: A survey and challenges. IEEE Commun. Surv. \& Tutorials 17, 1885-1922 (2015).

2. Jameel, F., Hamid, Z., Jabeen, F., Zeadally, S. \& Javed, M. A. A survey of device-to-device communications: Research issues and challenges. IEEE Commun. Surv. \& Tutorials 20, 2133-2168 (2018).

3. Wei, L., Hu, R. Q., He, T. \& Qian, Y. Device-to-device (d2d) communications underlaying mu-mimo cellular networks. In 2013 IEEE Global Communications Conference (GLOBECOM), 4902-4907 (IEEE, 2013).

4. Wang, L. \& Wu, H. Fast pairing of device-to-device link underlay for spectrum sharing with cellular users. IEEE Commun. Lett. 18, 1803-1806 (2014).

5. Gu, Y., Zhang, Y., Pan, M. \& Han, Z. Cheating in matching of device to device pairs in cellular networks. In 2014 IEEE Global Communications Conference, 4910-4915 (IEEE, 2014).

6. Wang, L. \& Stuber, G. L. Pairing for resource sharing in cellular device-to-device underlays. IEEE Netw. 30, 122-128 (2016).

7. Li, Y., Song, C., Jin, D. \& Chen, S. A dynamic graph optimization framework for multihop device-to-device communication underlaying cellular networks. IEEE Wirel. Commun. 21, 52-61 (2014).

8. Choi, K. W. \& Han, Z. Device-to-device discovery for proximity-based service in lte-advanced system. IEEE J. on Sel. Areas Commun. 33, 55-66 (2014).

9. Xu, C., Gao, C., Zhou, Z., Chang, Z. \& Jia, Y. Social network-based content delivery in device-to-device underlay cellular networks using matching theory. IEEE Access 5, 924-937 (2016).

10. Miao, L., Bai, B. \& Chen, W. 4-dmwm approach for caching based optimal d2d pairing and channel allocation: Centralized and distributed algorithm design. IEEE Access 4, 9213-9224 (2016).

11. Pourmoslemi, A., Rajabi, S. \& Salimi, M. Selecting the best transmitter in wireless device-to-device communications using a fuzzy decision-making method. In International Online Conference on Intelligent Decision Science, 509-520 (Springer, 2020).

12. Rajabi, S., Ghorashi, S. \& Shah-Mansouri, V. Impact of connecting to the nth nearest node in dedicated device-to-device communications. Electron. Lett. 54, 535-537 (2018).

13. Rashed, S. K., Asvadi, R., Rajabi, S., Ghorashi, S. A. \& Martini, M. G. Power allocation for d2d communications using max-min message-passing algorithm. IEEE Transactions on Veh. Technol. 69, 8443-8458 (2020).

14. Zadeh, L. A. Fuzzy sets. Inf. control 8, 338-353 (1965).

15. Cho, Y. J., Rassias, T. M. \& Saadati, R. Fuzzy operator theory in mathematical analysis (Springer, 2018).

16. Bellman, R., Kalaba, R. \& Zadeh, L. Abstraction and pattern classification. J. Math. Analysis Appl. 13, 1-7 (1966).

17. Bellman, R. E. \& Zadeh, L. A. Decision-making in a fuzzy environment. Manag. science 17, B-141 (1970).

18. Zadeh, L. A. Fuzzy sets and their application to pattern classification and clustering analysis. In Classification and clustering, 251-299 (Elsevier, 1977).

19. Bezdek, J. C. Fuzzy mathematics in pattern classification. Ph. D. Diss. Appl. Math. Cornell Univ. (1973).

20. Dunn, J. C. A fuzzy relative of the isodata process and its use in detecting compact well-separated clusters. (1973).

21. Mamdani, E. H. \& Assilian, S. An experiment in linguistic synthesis with a fuzzy logic controller. Int. journal man-machine studies 7, 1-13 (1975). 
22. Kosko, B. Neural networks and fuzzy systems: a dynamical systems approach to machine intelligence. QA76. 76 . E95 K86 (1992).

23. Ahvar, E., Pourmoslemi, A. \& Piran, M. J. Fear: A fuzzy-based energy-aware routing protocol for wireless sensor networks. arXiv preprint arXiv:1108.2777 (2011).

24. Bělohlávek, R., Dauben, J. W. \& Klir, G. J. Fuzzy logic and mathematics: a historical perspective (Oxford University Press, 2017).

25. Wei, W., Li, Z., Zhuoming, L. \& Xuejun, S. Fuzzy logic power control of device to device communication underlay td-lte-a system. In 2013 3rd International Conference on Consumer Electronics, Communications and Networks, 320-323 (IEEE, 2013).

26. Zhuoming, L. et al. Fuzzy mathematics and game theory based d2d multicast network construction. J. Syst. Eng. Electron. 30, 13-21 (2019).

27. Liu, M., Liu, Y., Zhou, Y., Zhang, W. \& Yu, Y. Research on d2d resource allocation algorithm based on improved fuzzy clustering. In Tenth International Conference on Digital Image Processing (ICDIP 2018), vol. 10806, 108066G (International Society for Optics and Photonics, 2018).

28. Chen, P., Xue, J., Chen, Y., Wei, S. \& Ji, Y. Resource allocation algorithm based on fuzzy cluster grouping for device-todevice communication. In Bio-Inspired Computing-Theories and Applications, 33-44 (Springer, 2015).

29. Subramani, M. \& Kumaravelu, V. B. A quality-aware fuzzy-logic-based vertical handover decision algorithm for device-todevice communication. Arab. J. for Sci. Eng. 44, 2413-2425 (2019).

30. Klement, E. P., Mesiar, R. \& Pap, E. Triangular norms, vol. 8 (Springer Science \& Business Media, 2013).

31. Schweizer, B. Associative funcrions and statistical triangle inequalities. Puble. Math. Debrecen 8, 169-186 (1961).

32. Munir, M., Kalsoom, H., Ullah, K., Mahmood, T. \& Chu, Y.-M. T-spherical fuzzy einstein hybrid aggregation operators and their applications in multi-attribute decision making problems. Symmetry 12, 365 (2020).

33. Wei, G. \& Zhao, X. Induced hesitant interval-valued fuzzy einstein aggregation operators and their application to multiple attribute decision making. J. Intell. \& Fuzzy Syst. 24, 789-803 (2013).

34. Zhao, X. \& Wei, G. Some intuitionistic fuzzy einstein hybrid aggregation operators and their application to multiple attribute decision making. Knowledge-Based Syst. 37, 472-479 (2013). 